\title{
Doppelheterodyn-Interferometrie für hochgenaue Vermessung im Nahbereich
}

\author{
E. Fischer, E. Dalhoff, T. Ittner, Z. Sodnik, H.J. Tiziani \\ Universität Stuttgart, Institut für Technische Optik, Pfaffenwaldring 9, D- 7000 Stuttgart 80
}

\section{Einleitung}

Für die berührungslose Entfernungsmessung bieten sich verschiedene optische Verfahren an. $\mathrm{Zu}$ den nichtkohärenten Methoden zāhlen die Triangulation /1,2,3,4/, Moire-, Laufzeit- und Phasenmeßtechniken. Geht man von einer Meßdistanz von $100 \mathrm{~m}$ und einer geforderten Auflösung von $0.1 \mathrm{~mm}$ aus, so erweisen sich die oben erwähnten Verfahren als nicht geeignet: Die Triangulation und die Moirê-Technik benötigt für die genannten Anforderungen eine zu große Basis, was einen kompakten Aufbau ausschlieBt. Bei der Laufzeitmessung und der inkohărenten PhasenmeBtechnik liegen die derzeit erzielbaren Auflősungen im Millimeterbereich. Eine Verbesserung der Auflösung erfordert einen großen elektronischen Aufwand.

Erweitert man die Verfahren zur Abstandsmessung um die kohărenten, so ist zunăchst die klassische Interferometrie zu nennen, bei der durch die Überlagerung zweier Wellenfelder ein Intensitātsmuster entsteht, aus welchem die Abstandsinformation gewonnen werden kann. Dabei ist es jedoch nicht mōglich, die Meßdistanz absolut zu ermitteln, da der Eindeutigkeitsbereich lediglich der halben Wellenlänge des Lichtes gleichkommt. Außerdem sind entsprechend der kurzen Wellenlānge die Stabilitātsanforderungen zu hoch, und es kőnnen lediglich Oberflächen mit optischer Qualităt vermessen werden, da andernfalls die entstehenden Speckles eine Auswertung erschweren oder gar unmōglich machen $16 /$.

Um die hohe Genauigkeit, die die interferometrische Meßtechnik bietet, auch an technischen Oberflächen nutzen zu kőnnen, muß auf grőßere Wellenlängen übergegangen werden. Ein möglicher Weg hin zu diesem Ziel stellt die Verwendung zweier nah beieinander liegender Wellenlängen dar. Diese sogenannte Zweiwellenlängen-Interferometrie reduziert die Empfindlichkeit soweit $7,8,9,10,11$, da 3 sie nunmehr einer effektiven Wellenlänge $\Lambda$ entspricht, die sich wie folgt berechnet $\Lambda=\lambda_{1} \cdot \lambda_{2} /\left|\lambda_{1}-\lambda_{2}\right|, \lambda_{1}$ und $\lambda_{2}$ sind die ursprünglichen Lichtwellenlängen. Kombiniert man die oben beschriebene Zweiwellenlängen-Interferometrie mit der Heterodyn-Methode, so ist man in der Lage, Entfernungen absolut zu messen, sowie optisch rauhe Objektoberflächen zuzulassen. Untersuchungen in dieser Richtung wurden bereits von Vry et al. und Kikuta et al. vorgenommen $/ 12,13 /$.

Im folgenden beschreiben wir den Aufbau und die Wirkungsweise eines sogenannten DoppelheterodynInterferometers, welches prinzipiell aus zwei unabhāngig voneinander arbeitenden Heterodyn-Interferometem besteht, die sowohl mit unterschiedlichen Lichtwellenlāngen $\lambda_{1}$ und $\lambda_{2}$ als auch mit unterschiedlichen Heterodyn-Frequenzen $f_{1}$ und $f_{2}$ arbeiten. Die Phase des Schwebungssignals mit der Frequenz $f_{1}-f_{2}$ hängt ab von der effektiven Wellenlänge $\Lambda$ und kann wie von Dändliker et al. gezeigt, zur Ermittelung der Entfernung ausgewertet werden /14/.

\section{Doppelheterodyn-Interferometer mit angepaßtem Bcugungsgitter}

Es wurde ein Doppelheterodyn-Interferometer (DHI) realisiert, mit dessen Hilfe es möglich war, erste Profilmessugen an technischen Oberflāchen sowie Untersuchungen verschiedenster Art durchzuführen. Bild 1 zeigt eine Skizze des verwendeten Aufbaus. Als Laserquelle diente ein kommerzieller Helium-Neon-Laser ( $\mathrm{HeNe}$ ), der so abgestimmt werden kann, daß gleichzeitig zwei Laserlinien emittiert werden $\lambda_{1}=633 \mathrm{~nm}, \lambda_{2}=640$ $\mathrm{nm})$. Die mit diesem Laser erzielbare synthetische Wellenlānge beträgt $55,5 \mu \mathrm{m}$, dies entspricht bei interferometrischen Messungen einem Eindeutigkeitsbereich von $27.8 \mu \mathrm{m}$.

Die Frequenzverschiebung in den beiden Interferometerreferenzarmen wird mittels cines $A O M\left(f_{d}=40\right.$ $\mathrm{MHz}$ ) und einer angepaßten Beugungsgitteranordnung durchgefüht. Bild 1 zeigt eine Skizze des Systems. Nach dcm Passieren des AOM schwingt das Licht in der ersten Beugungsordnung mit den Frequenzen $\nu_{1}+f_{d}$ und $\eta_{2}+f_{d}$. Eine notwendige Voraussetzung bei der Doppelheterodyn-Interferometrie ist, daß die beiden ein- 
zelnen Heterodyn-Interferometer auf verschiedenen Heterodyn-Frequenzen betrieben werden, da die Signalauswertung auf der Superheterodyn-Frequenz erfolgt. Aus diesem Grund ist eine zweite Frequenzverschiebung notwendig, die nur eine der beiden Lichtwellenlängen beeinflußt. Diese zweite Frequenzverschiebung erfolgt mittels einer Kombination von zwei Beugungsgittern.

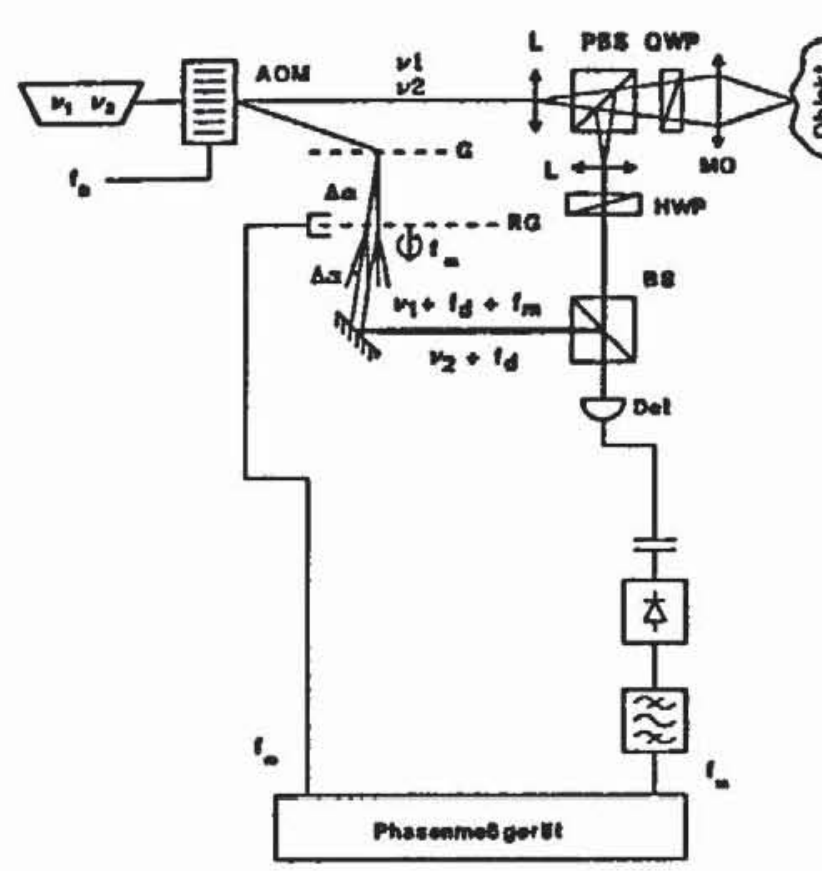

Bild 1: Skizze des HeNe-DHI Systems

Das aus dem $A O M$ in der ersten Beugungsordnung austretende Licht triff zunächst auf ein Beugungsgitter mit einer Gitterkonstanten $p_{1}\left(1 / p_{1}=600 \mathrm{Lp} / \mathrm{mm}\right)$. Aufgrund der hohen Ortsfrequenz des Gitters erfolgt im abgebeugten Licht eine chromatische Aufspaltung. In der ersten Beugungsordnung entsteht somit zwischen den beiden Lichtstrahlen mit $\lambda_{1}$ und $\lambda_{2}$ ein Differenzwinkel $\Delta \alpha$ (s. Bild 1). Direkt nach dem ersten Beugungsgitter befindet sich ein zweites Gitter mit der Gitterkonstanten $\mathrm{p}_{2}$, wobei $\mathrm{p}_{2}$ so gewăhit ist, daß für eine mittlere Wellenlănge $\lambda=\left(\lambda_{1}+\lambda_{2}\right) / 2$ der $A b$ lenkwinkel zwischen der nullten und der ersten Beugungsordnung gleich $\Delta \alpha$ wird. Nach dem Durchtritt durch das erste Beugungsgitter werden die in die erste Beugungsordnung mit den Differenzwinkeln $\Delta \alpha$ abgelenkten beiden Strahlen mit $\lambda_{1}$ bzw. $\lambda_{2}$ auf das zweite Beugungsgitter gelenkt. Hinter dem zweiten Gitter ergeben sich somit u.a. vier Teilstrahlen (s. Bild 1), Die jeweils paarweise parallel sind. Rotiert das zweite Beugungsgitter, so wird das Licht in den ersten Beugungsordnungen um den Betrag $f_{m}$ frequenzverschoben.

Das am MeBobjekt gestreute Objektlicht wird von der Beobachtungsoptik gesammelt, nach dem Strahlteiler BS (vgl. Bild 1) ergibt sich die Interferenz mit der jeweiligen Referenzwelle. Nach der Demodulation des amplitudenmodulierten Detektorsignales ergibt sich

$$
u(t)=U_{0} \cdot \cos \left(2 \pi \cdot\left(f_{2}-f_{1}\right) \cdot t-4 \pi \cdot z / \Lambda+\phi_{0}\right)
$$

dieses Meßsignal wird einem Phasenmeßgerāt zugefuhrt. Dieses wiederum bezieht sein Referenzsignal von einem Winkelschrittgeber, der an dem rotierenden Beugungsgitter angebracht ist.

Bild 2: Wiederholungsmessungen mit dem HeNe-DHI System an einer gefrästen Aluminiumprobe. Es sind Stufen mit $5 \mu \mathrm{m}$ und $10 \mu \mathrm{m}$ eingearbeittet.

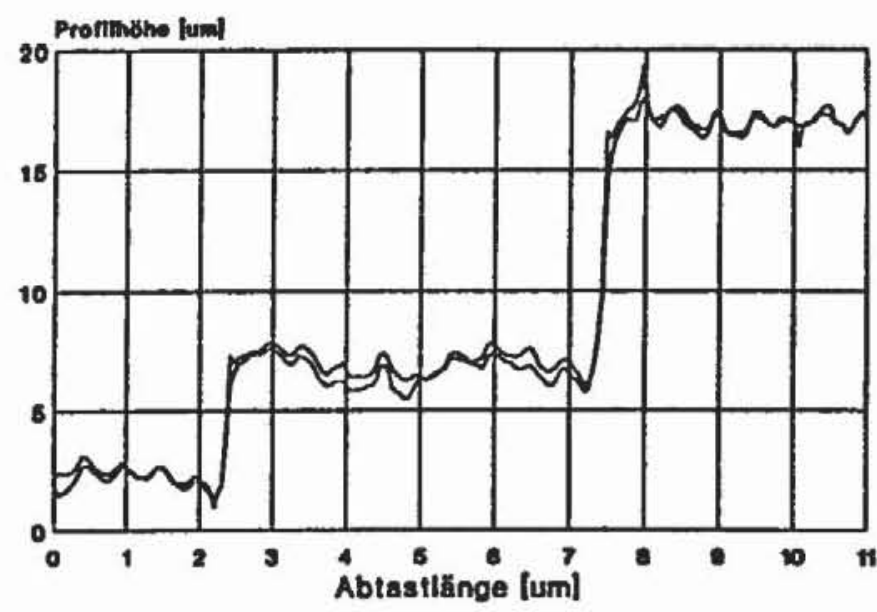

Im Bild 2 ist ein typisches Meßergebnis dargestellt, das mit dem HeNe-Doppelheterodyn-Interferometer erzielt wurde. Das Bild zeigt Profilmessungen an einer gefrästen Aluminiumprobe, in der Stufen mit $5 \mu \mathrm{m}$ und $10 \mu \mathrm{m}$ eingearbeitet sind. Das Meßsystem löst diese Stufen eindeutig auf. Es sind zwei Messungen darge- 
stellt, die im Abstand von mehreren Minuten aufgenommen wurden. Die Meßunsicherheit des Systemes für Wiederholungsmessungen liegt bei $0.5 \mu \mathrm{m}$. Die geringe Differenz zwischen den beiden Messungen ist eine:seits durch Umwelteinflüsse (Temperaturschwankungen etc.) und andererseits durch systembedingte MeBunsicherheiten (rotierendes Gitter etc.) bedingt.

\section{Einfluß der Anzahl der in der Detektorapertur befindlichen Speckle}

Das Verhăltnis zwischen dem Detektordurchmesser und der durchschnittlichen Specklegrōße beeinflußt zwei Eigenschaften des Messignals: Seinen Rauschabstand und den statistischen Phasenfehler.

1. Die Phase innerhalb eines voll entwickelten Specklefeldes ist gleichverteilt /15/. Liegen also mehrere Speckle innerhalb der Detektorapertur, so werden die zugehōrigen Heterodynsignalbeitrăge i.d.R. nicht gleichphasig schwingen. Aufgrund der Integration des Detektors über die Signale der in seiner Apertur befindlichen Speckle ergibt sich eine Abschwãchung des Signals, die mit der Zahl der Speckle zunimmt. Als Maß hierfür kann der sogenannte modifizierte Mischerkoeffizient gelten, ein allgemeines MaB für den zeitlichen Kontrast des resultierenden Heterodynsignals /16/. Bereits bei einem der durchschnittlichen SpecklegröBe entsprechenden Detektordurchmesser wird der Mischerkoeffizient gegenüber demjenigen des Punktdetektors etwa auf ein Fünftel reduziert; dies fūhrt zu einer quadratischen Absenkung des Rauschabstandes. Es läBt sich zeigen, daß die Signalausfallswahrscheinlichkeit, einen beliebigen Schwellwert für den Rauschabstand vorausgesetzt, mit zunehmender Specklezahl in der Detektorapertur zunimmt.

2. Aufgrund der Dekorrelation zwischen den beiden das Doppelheterodynsignal generierenden Specklefeldern der Wellenlängen $\lambda_{1}, \lambda_{2}$ ergibt sich für das Messignal ein statistischer Phasenfehler, der für einen Punktdetektor theoretisch und experimentell analysiert wurde /12/. Mittelt der Detektor über die Heterodynsignale mehrerer Speckle, so findet eine Mittelung über den Phasenfehler statt. Dies führt zu einer Verbesserung der Wahrscheinlichkeitsverteilung des Phasenfehlers mit zunehmender Zahl der Speckle in der Detektorapertur. Abbildung 3 zeigt experimentell aufgenommene Verteilungskurven für drei verschiedene Detektorgrößen.

Bild 3: Experimentell aufgenommene Wahrscheinlichkeitskurven des statistischen Phasenfehlers für drei verschiedene Detektorgrößen

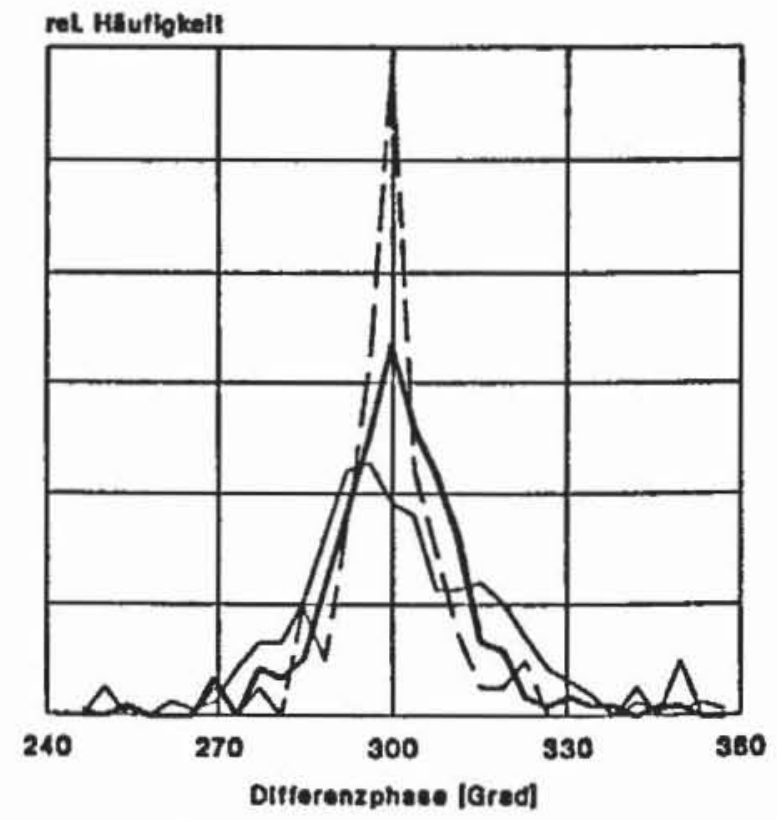

Detektordurchmesser

- 120 um 270 um $---900 \mathrm{um}$

durchechnittL. Specklegrose 135 ve

Eine Betrachtung des Rauschabstands legt also nahe, den Detektor so klein wie möglich zu wählen, eine Betrachtung des statistischen Phasenfehlers dagegen fordert möglichst viele Speckle im Detektor. Je nach den Parametern des Messystems und der Messaufgabe (Schwebungswellenlänge, Meßintegrationszeit, Obernächenrauhigkeit, Meßentfernung etc.) ist hier ein Optimum zu wählen. 


\section{Doppelheterodyn-Interferometer für den Nahbereich}

Nachdem, wie im vorstehenden Kapitel beschrieben, gezeigt werden konnte, daß sich die DoppelheterodynInterferometrie für Profil- bzw. Abstandsmessungen eignet, wird dieses Verfahren weiterentwickelt werden für den Nahbereich. Hierfür ist es notwendig, die synthetische Wellenlänge des DHI-Systemes veränderbar zu gestalten, um das Verfahren für hochgenaue Messungen einzusetzen. Die maximal erreichbare Meßgenauigkeit hăngt dabei entscheidend von der Stabilität der Differenzfrequenz der zwei verwendeten Lichtfrequenzen ab. Wird eine Laserquelle benutzt, die im Monomodebetrieb arbeitet (z.B. strom- und temperaturstabilisierte Laserdiode) und zur Generienung der zweiten Lichtwellenlänge ein ultra-hochfrequenter akustooptischer Frequenzschieber (UHF-AOM) mit einer Beugungseffizienz von $50 \%$, so läBt sich die synthetische Wellenlănge durch Ändem der UHF-AOM Ansteuerfrequenz sehr einfach variieren. Die Stabilităt der synthetischen Wellenlänge wird dabei durch die Frequenzstabilităt des AOM bestimmt. Kommerziell verfügbare AOM Systeme besitzen Frequenzstabilitãten, die den Anforderungen genūgen. Da bei der Realisierung des geplanten DHI Systemes die beiden benutzten Einzelheterodyn-Interferometer z.T. getrennte Wege durchlaufen mũssen, besteht die Notwendigkeit, ein Referenzinterferometer in das System zu integrieren.

as Doppelheterodyn-Interferometers befindet sich derzeit im Aufbau. Subsysteme wurden bereits ausgetestet. Als Laserquelle kommt eine strom- und temperatutstabilisierte Laserdiode mit einer maximalen Ausgangsleistung von $30 \mathrm{~mW}$ zum Einsatz. Nachdem das Licht den UHF-AOM verlassen hat, sind zwei gleich starke Lichtstrahlen vorhanden, deren Lichtfrequenzen $500 \mathrm{MHz}$ auseinander liegen. Somit steht ein Eindeutigkeitsbereich für Abstandsmessungen von $30 \mathrm{~cm}$ zur Verfügung. Nachdem die UHF-Treiberfrequenz auf z.B $501.5 \mathrm{MHz}$ umgeschaltet wird, lāßt sich eine erneute Messung durchführen, jetzt aber mit einer neven synthetischen Wellenlănge. Aus der Kombination der beiden Einzelmessungen /17/ ergibt sich somit ein resultierender Eindeutigkeitsbereich von $100 \mathrm{~m}$.

Von den beiden den UHF-AOM verlassenden Lichtstrahlen wird ein Teil der Energie als Referenzstrahl abgespalten und in jeweils einem weiteren AOM um $80 \mathrm{MHz}$ bzw. $80.1 \mathrm{MHz}$ frequenzverschoben, so daß sich eine Superheterodynfrequenz von $100 \mathrm{KHz}$ ergibt. Die weitere Strahlfūhrung erfolgt in einem Glasfasernetzwerk, um einen mōglichst kompakten Aufbau zu realisieren. Bei der Sende- und Empfangsoptik wird eine gute Trennung zwischen den beiden Teilstrahlengängen angestrebt um etwaiges Übersprechen zu vermeiden.

\section{Zusammenfassung}

In diesem Aufsatz wurde ein Meßsystem vorgestellt, welches sich aus der Kombination der Zweiwellenlängen-Interferometrie mit dem intensitătsunabhăngigen Heterodyn-Verfahren zusammensetzt. Die resultierende Doppelheterodyn-Interferometrie vereinigt die Vorteile der beiden Verfahren. Ein großer Eindeutigkeitsbereich entsprechend der effektiven Wellenlänge $\Lambda$ entsteht, darüberhinaus sind optisch rauhe Oberflächen sind als Meßobjekte zulässig; reduzierte Empfindlichkeit gegenüber Vibrationen und Umwelteinflüssen, hohe Auflōsung infolge elektronischer Phasenbestimmung sowic hochgenaue Abstandsmessung in Echtzeit sind weitere Vorteile.

Das Doppelheterodyn-Interferometer wird in Zukunft zu einem effektiven und dynamischen MeBinstrument zur hochgenauen Entfernungsmessung im Mikrometerbereich bis hin zu Meßdistanzen von $100 \mathrm{~m}$ entwickelt werden, für das sich schon jetzt zahlreiche Einsatzmöglichkeiten abzeichnen.

\section{Literatur:}

III Bickel, G. et al.: Triangulation with expanded range of depth

Optical Engineering 24, 975, (1985)

12/ Rioux, M.: Laser range finder based on synchronized scanners

Applied Optics 23, 3837, (1984)

13/ Hảusler, G. et al.: Telecentric scanner for 3-D sensing

Optical Engineering 24, 978, (1985)

14/ Seitz, G. et al.: 3-D-Koordinatenmessung durch optische Triangulation

Feinwerktechnik \& Meßtechnik 94, 423, (1986)

15I Greve, A.; Harth, W.: Laser diode distance meter in a KERN DKM 3A theodolite

Applied Optics 23, 2982, (1984) 
16/ Tiziani, H.J.: Rechnerunterstūtzte Laser-MeBtechnik Technisches Messen 54, 221, (1987)

171 Tiziani, H.J.: Optical methods for precision measurements Optical and Quantum Electronics 21, 253, (1989)

18/ Wyant, J.C.: Testing aspherics using two-wavelength holography Applied Optics 10, 2113, (1971)

19/ Polhenus, C.: Two-wavelength interferometry Applied Optics 12, 2071, (1973)

I10/ Küchel, M.; Tiziani, H.J.: Real-time contour holography using BSO crystals Optics Communications 38, 17, (1981)

111/ Creath, K. et al.: Contouring aspheric swurfaces using two-wavelength phase- shifting interferometry, Optica Acta 32, 1455, (1985)

112/ Vry, U.; Fercher A.F.: Higher Order Statistical Properties of Speckle Fields and their Application to Rough Surface Interferometry ,JOSA A Vol.3, 988, (1986)

113/ Kikuta, H. et al.: Distance measurement by the wave length shif of laser diode light, Applied Optics 25, 2976, (1986)

114/ Danndliker, R. et al.: Two wavelength laser interferometry using super-heterodyne detection Proceedings of ICO 14 Congress, Quebec, Canada, paper B 1.2, (1987)

$115 /$ Dainty J. C. (Ed.); Laser Speckle and Related Phenomena, Springer Verlag Berlin, (1979), Kap. 2: Goodman J.W. Statistical Properties of Laser Speckle Patterns

116/ Dalhoff E.; Untersuchung der Sensibilitāt des Doppelheterodynsignals gegenüber der Granulation, Diplomarbeit Universität Stuttgart, Institut für Technische Optik, (1991)

117 Hart, P.: Fernwirktechnik der Raumfahrt Springer Verlag Berlin, Heidelberg, 2. Auflage, (1988) 